\title{
Two Successful Pregnancies in a Woman without Kidneys: A Case Report
}

\author{
Anna Curtis ${ }^{1 *}$, Christian Lamb ${ }^{2}$, Sarah Atallah ${ }^{1}$, Saras Singh ${ }^{1}$, and Amit Patel ${ }^{3}$ \\ ${ }^{1}$ School of Medicine, University of Missouri-Kansas City, Kansas City, USA \\ ${ }^{2}$ Department of Medicine, Brooke Army Medical Center, San Antonio, Texas, USA \\ ${ }^{3}$ Truman Medical Center, Kansas City, USA
}

\begin{abstract}
Background: Bilateral nephrectomy is a rarely used approach to control refractory hypertension in patients with anuric end-stage renal disease (ESRD) who already require dialysis. This procedure results in complete dependence on hemo- or peritoneal dialysis to maintain metabolic homeostasis and has fallen out of favor given advancements in anti-hypertensive pharmacotherapy. Given the rarity of this management decision, there is a paucity of scientific literature regarding how the absence of kidneys impacts childbearing. On review of the literature regarding bilateral nephrectomy and pregnancy, only one case report was identified. That article, published in 2013, details a woman receiving intermittent peritoneal dialysis who successfully carried a pregnancy to 29 weeks, with the birth of a healthy child. We present the first known case of successful pregnancy in a woman on intermittent hemodialysis without kidneys. Additionally, the patient had two successful pregnancies and was receiving biweekly eculizumab infusions for a diagnosis of atypical hemolytic uremic syndrome (aHUS).

Case presentation: A 35-year-old multiparous female with hypertension and end-stage renal disease secondary to atypical hemolytic uremic syndrome (aHUS) on chronic intermittent hemodialysis and biweekly eculizumab infusion underwent bilateral nephrectomy for resistant hypertension. Two years following nephrectomy, the patient presented to an emergency department (ED) and was found to be pregnant at 21 weeks gestation. The patient gave birth vaginally at 27 weeks gestation to a small for gestational age, but otherwise healthy infant. Two years following delivery of her first pregnancy and four years following bilateral nephrectomy, the patient was found to again be pregnant at 9 weeks gestation. She delivered via cesarean section at 23 weeks gestation due to preeclampsia. The patient and her child did well postpartum.
\end{abstract}

Conclusion: We report the first known case of successful pregnancies on hemodialysis after bilateral nephrectomy.

\section{Introduction}

Bilateral nephrectomy is a controversial mode of blood pressure control in those with malignant hypertension and anuric end-stage renal disease (ESRD). Theoretically, this procedure modulates the renin-aldosterone-angiotensin system (RAAS) by removing the kidneys and decreasing serum renin among other mechanisms. Unfortunately, this approach has dropped out of favor in light of advancements in dialysis and pharmacologic therapies $[1,2]$. Occasionally, there are patients with malignant hypertension that do not respond to aggressive pharmacologic, dietary, and dialytic therapy, resulting in extensive end-organ damage and shortened life span. For these patients, bilateral nephrectomy with subsequent renal transplantation may be their only option for improved prognosis and quality-of-life [1].

Pregnancy in patients following bilateral nephrectomy is sparsely reported. Currently, only a single case report published in 2013 details such an account. According to the arti- cle, a woman receiving intermittent peritoneal dialysis carried a successful pregnancy following bilateral nephrectomy performed for bilateral pyelonephritis [3]. No other reports of pregnancy following bilateral nephrectomy were found. With such a paucity of reports, obstetricians and nephrologists have few resources available regarding the prevalence, maternal/neonatal mortality, and complications that may occur in this specific patient population.

*Corresponding author: Anna Curtis, Division of Nephrology, Department of Medicine, School of Medicine, University of Missouri-Kansas City, Kansas City, MO 64108, USA

Accepted: October 17, 2020

Published online: October 19, 2020

Citation: Curtis A, Lamb C, Atallah S, et al. (2020) Two Successful Pregnancies in a Woman without Kidneys: A Case Report. Ann Nephrol 5(1):75-77 
We present the first case of successful pregnancy in a woman who was receiving intermittent hemodialysis following bilateral nephrectomy. The bilateral nephrectomy had been performed to curtail her malignant hypertension. The management, complications, and other novel aspects of this case are discussed herein.

\section{Case Presentation}

A 35-year-old multiparous female with hypertension presented to the emergency department (ED) at 22 weeks gestation with complaints of abdominal pain, lower extremity edema, and anuria. Laboratory studies were consistent with acute renal failure and the patient received emergent hemodialysis. Further diagnostic work-up revealed atypical hemolytic uremic syndrome (aHUS) and she was started on biweekly eculizumab infusions.

Following discharge, she remained anuric and continued regular hemodialysis three times weekly. She delivered that pregnancy 26 weeks gestation via emergent cesarean section for non-reassuring fetal heart tones and breech positioning. Ultimately, the infant was small for gestational age, but was discharged from the hospital after a short stay in the Neonatal Intensive Care Unit (NICU).

Seven months later the patient underwent bilateral laparoscopic nephrectomy with expectations to receive a renal transplant. Attempts to wean eculizumab infusions were unsuccessful and resulted in aHUS flares. She was ultimately removed from the renal transplant registry.

Two years following nephrectomy, the patient presented to an outside ED and was found to have a positive $\beta$-HCG. Ultrasound confirmed a 21 week gestation. She ultimately required hemodialysis six times weekly with an average ultrafiltrate of $2.5 \mathrm{~L}$ each session. Her pregnancy was complicated by pre-eclampsia. At 27 weeks gestation the patient again presented to our facility's ED in labor and vaginally delivered a small for gestational age, but otherwise healthy infant. The infant was discharged from the NICU after a short stay. Postpartum, the patient's dialysis schedule returned to three times weekly.

Two years following delivery of her second pregnancy, the patient was found to again be pregnant at 9 weeks gestation. She was transitioned to dialysis six days weekly with an average ultrafiltrate of $2 \mathrm{~L}$ per session. Chromosomal analysis revealed monosomy $X$ in the fetus. She delivered via cesarean section at 23 weeks gestation due to preeclampsia. The patient and her child did well postpartum.

\section{Discussion}

Currently, no literature exists detailing the barriers to conception, antepartum management, and postpartum outcomes in patients who have undergone bilateral nephrectomy and wish to become pregnant. The case described herein is the only known report of a woman without kidneys receiving chronic hemodialysis who became pregnant and delivered two healthy infants. While no studies exist in this unique patient population, there is ample data regarding women with ESRD who become pregnant. Therefore pregnancy in ESRD, as well as the previously mentioned case of a female without kidneys who became pregnant while receiving peritoneal dialysis will be discussed herein.

Women with ESRD receiving hemodialysis are at a significant disadvantage for childbearing compared to their healthy counterparts. As the glomerular filtration rate declines, uremia, hyperprolactinemia, and altered sex hormone synthesis impairs a multitude of organ systems, leading to menstrual irregularities, an ovulation and impaired libido. Studies have revealed that pregnancies occur in just $1-7 \%$ of women on hemodialysis. For those who successfully conceive, only $80 \%$ have live infants at birth [4]. Despite these harsh environments for conception and development, our ESRD patient was able to conceive and carry two pregnancies following bilateral nephrectomy.

While no studies exist regarding the impact of bilateral nephrectomy on pregnancy, recommendations regarding the management of pregnancy in ESRD have been established. These guidelines suggest increasing the frequency of peritoneal or hemodialysis sessions, dietary modifications, and altering pharmacotherapy $[5,6]$. As seen in the report by AbuZeid, et al., the patient's peritoneal dialysis was increased from 18 hours per week to 22 hours per week following the discovery of her pregnancy [3]. Similarly, the patient featured in this case report had her hemodialysis frequency increased from 3 times to 6 times weekly, with an averaged ultrafiltrate of $2.5 \mathrm{~L}$ per hemodialysis session.

Currently, there is no evidence available to guide ultrafiltrate in pregnant women on hemodialysis or in those without kidneys. Ultrafiltrate removal is a clinical determination based on a patient's dry weight and clinical status, which can be challenging in pregnancy. Normal pregnancy is associated with 11-16 kg of healthy weight-gain which can vary significantly between individuals, making the estimation of difficult. Additionally, the aggressive removal of ultrafiltrate may be associated with placental ischemic injury and fetal distress. Therefore, multidisciplinary care from both fields of maternal-fetal-medicine and nephrology may be essential in guiding ultrafiltration and hemodynamic homeostasis of both mother and fetus [7].

Unfortunately, despite following the above guidelines and closely monitoring ultrafiltrate removal, both of our patient's pregnancies were complicated by pre-eclampsia, small for gestational age, and preterm birth. Similarly, the pregnancy reported by Abu-Zeid, et al. was also complicated by these same issues. Both of these cases highlight the unique challenges in managing pregnancy in patients receiving hemodialysis [3].

Another interesting aspect of this case is the novel pharmacotherapy this patient received throughout her two pregnancies. Eculizumab is a monoclonal antibody approved by the Food and Drug Administration for the treatment of aHUS [8]. This thrombotic microangiopathy was the primary cause of ESRD in our patient, which was diagnosed prior to her bilateral nephrectomy and subsequent pregnancies. Currently, there no controller studies detailing the use of this pharmacotherapy in pregnancy. In lieu of this, multiple attempts at dis- 
continuing the agent were made but the patient was unable to tolerate cessation of the agent. For this reason, the patient continued eculizumab throughout the two pregnancies discussed in this report. Despite controlled studies demonstrating eculizumab safety in pregnancy, there is a growing host of new literature which suggests it may be safe in this patient population, but further research is required [9-11].

\section{References}

1. Zazgornik J, Biesenbach G, Janko O, et al. (1998) Bilateral nephrectomy: The best, but often overlooked, treatment for refractory hypertension in hemodialysis patients. American Journal of Hypertension 11: 1364-1370.

2. Macsim L, Stróżecki P, Miśkowiec-Wiśniewska I, et al. (2012) Bilateral nephrectomy as a rescue therapy for hemodialyzed patients with malignant hypertension - case report. Case Rep Nephrol Urol 2: 11-14.

3. Abu-Zaid A, Nazer A, Alomar O, et al. (2013) Successful pregnancy in a 31-year-old peritoneal dialysis patient with bilateral nephrectomy. Case Rep Obstet Gynecol 2013: 173405.

4. Tangren J, Nadel M, Hladunewich MA (2018) Pregnancy and end-stage renal disease. Blood Purif 45: 194-200.
5. Hladunewich M, Hou S, Odutayo A, et al. (2014) Intensive hemodialysis associates with improved pregnancy outcomes: a Canadian and United States cohort comparison. J Am Soc Nephro 25: 1103-1109.

6. Asamiya $Y$, Otsubo S, Matsuda Y, et al. (2009) The importance of low blood urea nitrogen levels in pregnant patients undergoing hemodialysis to optimize birth weight and gestational age. Kidney Int 75: 1217-1222.

7. Marques $L$ (2019) Pregnancy among women undergoing chronic hemodialysis: Effect of ultrafiltration during gestation. J Clin Nephrol Ther 3: 1-2.

8. (2007) Eculizumab. Alexion Pharmaceuticals, New Haven, CT, USA.

9. Ardissino G, Wally Ossola M, Maria Baffero G, et al. (2013) Eculizumab for atypical hemolytic uremic syndrome in pregnancy. Obstet Gynecol 122: 487-489.

10. Servais A, Devillard N, Frémeaux-Bacchi V, et al. (2016) Atypical haemolytic uraemic syndrome and pregnancy: outcome with ongoing eculizumab. Nephrol Dial Transplant 31: 2122-2130.

11. Sarno L, Tufano A, Maruotti G, et al. (2019) Eculizumab in pregnancy: A narrative overview. J Nephrol 32: 17-25.

DOI: $10.36959 / 832 / 403$

Copyright: (C) 2020 Curtis A, et al. This is an open-access article distributed under the terms of the Creative Commons Attribution License, which permits unrestricted use, distribution, and reproduction in any medium, provided the original author and source are credited. 